\title{
Sign of the dominant charge carriers in photorefractive crystals determined by a phase-locked holographic technique
}

\author{
A.A. Freschi ${ }^{a, *}$, F.A. Callegari ${ }^{a}$, F.S. De Vicente ${ }^{b}$, M.R.R. Gesualdi $^{a}$ \\ ${ }^{a}$ UFABC - Univ Federal do ABC, Centro de Engenharia (CECS), Avenida dos Estados 5001, 09210-170 Santo André, SP, Brazil \\ ${ }^{\mathrm{b}}$ UNESP, Univ Estadual Paulista, Departamento de Física (IGCE), Caixa Postal 178, 13500-970 Rio Claro, SP, Brazil
}

\section{A R T I C L E I N F O}

\section{Article history:}

Received 24 July 2015

Accepted 8 August 2015

Available online 14 August 2015

\section{Keywords:}

Photorefractive

Photoconductivity

Charge carrier

Hologram

Phase control

Feedback system

\begin{abstract}
A B S T R A C T
This work presents a holographic method based on active feedback techniques for determining the sign of the dominant charge carriers in photorefractive materials. A two-step procedure is proposed: first off a stationary phase-locked hologram is recorded; an electric field normal to the grating layers is then applied to the material, thus producing a running hologram. The sign of the charge carriers is determined by comparing the direction of the applied field with the direction of the hologram movement, which is known through the automatically attached light pattern. The method can be applied from highly photoconductive to highly insulating materials. Furthermore, no information on any material parameter is required. The method is validated by a set of holographic experiments using a $\mathrm{Bi}_{12} \mathrm{TiO}_{20}$ crystal that has electrons as the majority photocarriers.
\end{abstract}

(c) 2015 Elsevier B.V. All rights reserved.

\section{Introduction}

Photorefractive crystals are very well suited to record phase volume holograms by the exposition to a light interference pattern [1]. The record mechanism involves photoexcitation, migration and trapping of charge carriers (electrons and/or holes), resulting in a spatial redistribution of electric charge within the crystal volume. A space charge field arises, which in turn modulates the refractive index of the material through the linear electrooptic effect. An important feature of photorefractive materials is that they can be tailored to many different purposes, such as data storage, phase conjugation, optical filters, and signal processing, just to name a few [2]. This great variety of applications is only possible due to the large diversity of crystals developed during the last decades $[3,4]$. In addition, crystals have been doped with many types of impurities at different concentrations to provide adequate donor and acceptor centers of charge carriers; these impurities affect the photocarrier sign, among other properties.

The photocarrier sign is closely linked to the charge transport properties of the material and therefore of fundamental importance from the viewpoint of applications. Although usually unwanted, both electrons and holes may be photoexcited depending on the active donor centers. In any case, the sign of the dominant charge carriers depends not only on the host material and on

\footnotetext{
* Corresponding author.

E-mail address: agnaldo.freschi@ufabc.edu.br (A.A. Freschi).
}

doping, but also on the crystal's origin and on experimental conditions such as the illumination wavelength, light intensity, and grating period [3-5]. It is therefore very important to have access to reliable techniques for determining the photocarrier sign and the corresponding charge transport parameters; a fact that was recognized since the early days of the development of photorefractive materials. Some well known techniques that were adapted and applied to different crystals include: direct photoconductivity measurements [6], different approaches of energy transfer measurements based on holographic techniques [5,7-11], photoinduced Hall-current measurements [12,13], classical and holographic time-of-flight techniques [14,15], electron paramagnetic resonance spectroscopy [16], among others [17,18].

The method here presented is devoted to answer a simple question about the photoconductivity in a photorefractive material: are the dominant charge carriers electrons or holes ( $n$ or $p$ photoconductivity)? In its present form, it does not provide any additional information on other charge transport parameters, such as the carriers mobility, the diffusion (or drift) length, or the density of photoactive centers. However, a combination of distinct properties make this new method very attractive: (1) wide range of application, covering from highly photoconductive to highly insulating materials, (2) the method can be applied to crystals subjected to the most common internal charge driving forces (diffusion and bulk photovoltaic), which include both local and non-local holograms, (3) no prior knowledge on any material parameter is required (such as the sign of the electrooptic coefficient or the 
polarity of the $c$-axis in photovoltaic crystals), and (4) no need of several measurements; in fact, a single measurement is enough to answer unambiguously the proposed question.

\section{Theoretical principle of the method}

Consider the interference of two coherent beams in a photorefractive crystal. The development of the hologram is sketched in Fig. 1. The light intensity $(I)$ excites electrons into the conduction band (left column) or holes into the valence band (right column). The generation rate $(G)$ of free charge carriers is in phase to the light pattern. Three different mechanisms can be involved in the movement of the charge carriers: diffusion, the bulk photovoltaic effect, and drift (when an external electric field is applied). For the sake of simplicity, we neglect the bulk photovoltaic effect in Fig. 1; the generalization including photovoltaic currents (which are common for non-centrosymmetric crystals) will be discussed later at the end of the paper. Assume that initially no external field is applied to the sample. The existence of a concentration gradient causes the photoexcited carriers to diffuse from the bright areas to the dark areas before they recombine with the photorefractive trap centers. Thus, the diffusion gives rise to an amplitude difference between the generation and recombination $(R)$ rates, which means that positive and negative ionized immobile centers are distributed inside the crystal volume. The corresponding space charge density $(\rho)$ is the origin of the internal space charge electric field that modulates the refractive index of the material through the Pockels effect. The vertical dashed lines in Fig. 1 draw attention to the phase shift between the charge modulation $\rho$ and the light pattern $I$; it is 0 or $\pi$ depending on whether electrons or holes diffuse. It is worth noting that this difference on the phase shifts leads to
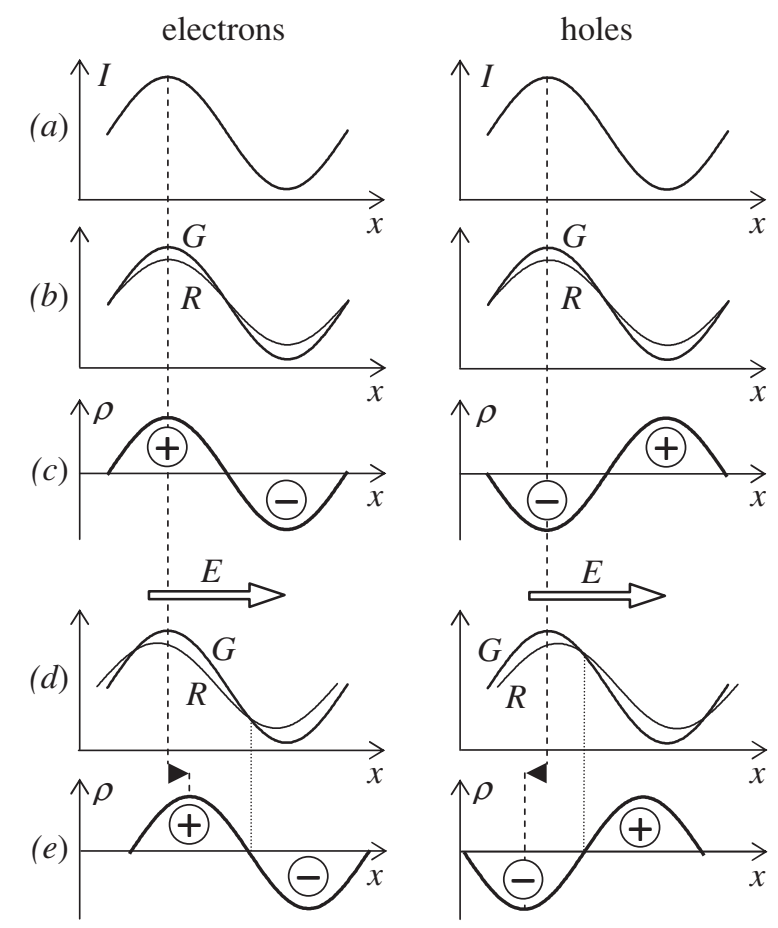

Fig. 1. Development of the photorefractive hologram. Charge carriers are assumed to be electrons in the left column and holes in the right one. The $x$ coordinate is oriented along the direction of the grating wave vector. (a) Intensity $(I)$ of the light interference pattern, (b) generation $(G)$ and recombination $(R)$ rates of free charge carriers under diffusion regime, (c) electric charge density $(\rho)$ due to the ionized donor/acceptor photorefractive centers, (d) generation and recombination rates of free charge carriers under the influence of a steady electric field $(E)$, and $(e)$ the corresponding electric charge density. When compared to the diffusion regime, the charge density is shifted to the right (same direction of the electric field) if the free carriers are electrons and to the left in the case of holes. different energy redistribution between the recording beams in two beam coupling experiments, which can be used to identify the photocarrier sign [7]. However, this information by itself does not always allow determining the type of charge carrier; for example, additional experiments (such as ellipsometric measurements) are required to give the sign of the electro-optic coefficient in cubic crystals such as those of the sillenite family $\left(\mathrm{Bi}_{12}(\mathrm{Ge}, \mathrm{Si}, \mathrm{Ti}) \mathrm{O}_{20}\right)$ [5]. Back to Fig. 1, assume now that a steady electric field $(E)$ is applied to the sample along the grating wave vector $\mathbf{K}$ ( $x$-direction). The diffusion is still present, but due to the different signs of the charge carriers, the electric force shifts the carriers in opposite directions depending on whether they are electrons or holes; in the figure, electrons move to left and holes to the right. The amplitude of the applied field is supposed to be small enough so as to shift the recombination rate by a small amount (short drift length of the carriers). As a result, the space charge density shifts to the right (same direction as the electric field $E$ ) if the carriers are electrons, or to the left (opposite to $E$ ) if they are holes. Contrary to what we might think, it is interesting to note that the space charge density (and hence the hologram), moves in opposite direction to that of the mobile charges.

\section{Experimental implementation}

In what follows, the experimental procedure on how to determine the direction of the hologram movement is described. The technique is based on the active phase control between the light pattern and the hologram being recorded. The general scheme of the interferometric setup used in the experiments is depicted in Fig. 2. The interference of two laser beams ( $R$ and $S$ ) generates a light pattern inside the crystal. A spatial modulation of the charge density grows up; it is accompanied by the internal space charge electric field and the refractive-index pattern (the hologram). Let us call $x$ the spatial coordinate along the direction of the grating wave vector. Because the recording light fulfills the Bragg condition, part of the incident light is diffracted from one recording beam into the other one. Therefore, each beam in each one of the two output directions is the superposition of a transmitted and a diffracted wave. The phase shift $\varphi$ between the interfering waves depends on the phase shift between the hologram and the light pattern (also called holographic phase shift), and on the beam coupling conditions between the $R$ and $S$ beams as they propagate

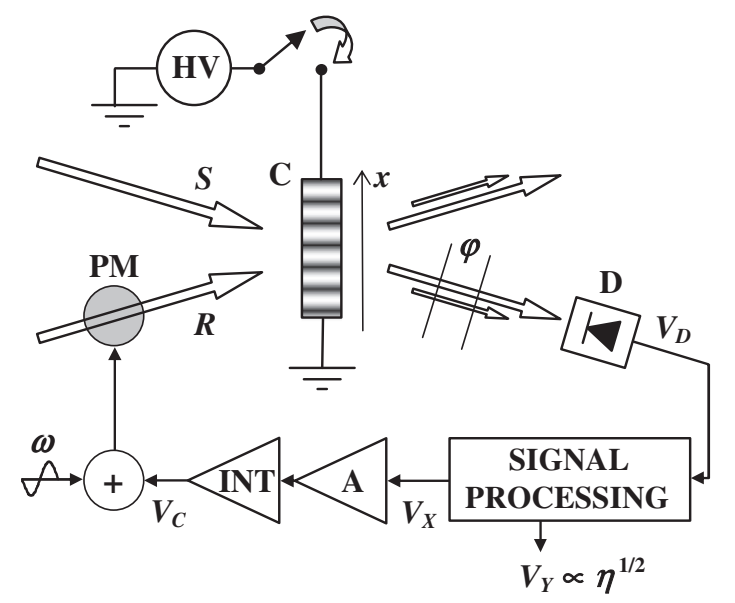

Fig. 2. General scheme of the holographic setup. HV: high voltage source, $C$ : photorefractive crystal, $R$ and $S$ : input light beams, PM: phase modulator, $\varphi$ : phase shift between the transmitted and diffracted beams, $D$ : photodetector, $V_{D}$ : photodetector's output voltage, $V_{X}$ and $V_{Y}$ : phase sensitive voltages generated by the signal processing circuit, A: amplifier, INT: integrator, $V_{C}$ : control voltage fed back to the interferometer, $\omega$ : weak and fast sinusoidal phase modulation (dither signal), $\eta$ : diffraction efficiency of the hologram. 
through the crystal volume $[19,20]$. The straight relationship between the holographic phase shift and the phase shift between the transmitted and diffracted waves opens the possibility to use the latter to control the spatial position of the light interference pattern relative to the hologram by means of an active feedback loop. The holographic recording in this condition is usually referred as self-stabilized, because the very hologram that is being recorded is used in real time as reference for stabilizing the holographic setup [21]. In the case of photorefractive materials, the phase value chosen for stabilization is of critical importance due to the dynamic nature of the photorefractive hologram [22,23]. In fact, if the phase $\varphi$ imposed by the feedback system is different from the one that arises naturally from a stationary (non-moving) light pattern, the net result of the process of trap recharging is a hologram that runs attached to the recording light pattern, like a cat chasing its tail [24]. In this case, the speed of the hologram is determined by the degree of phase mismatch, the photoconductivity of the crystal, and on other material and experimental parameters. In practice, the spatial position of the light pattern is controlled by means of a phase modulator (PM) placed in one of the arms of the interferometer. This control is usually done by a voltage signal $\left(V_{C}\right)$ generated by the feedback system and applied to the modulator. The two possible movement directions of the pattern along the $x$ coordinate (let us call them right and left) are related to the slope (positive or negative) of the control voltage $V_{C}$. In our setup, we used a microscope tube (focused on one edge of the crystal) to establish the relation between the voltage slope and the movement direction of the pattern. Throughout this text we define as positive the $x$-direction corresponding to a positive voltage slope; in this case, the light pattern is moving to the right when viewed against the direction of light propagation.

Several feedback techniques for stabilizing holographic setups have been reported in the literature; a general overview can be found in chapter 6 of Ref. [25], and more recent developments in [26-28]. In principle, the main goal of a feedback system is to generate an error signal suitable for the particularities of the experiment (desired phase shift, frequency bandwidth, etc.). Details on the feedback system used in our experiments are described in Ref. [28]. The choice was simply a matter of convenience, but different techniques could be applied as well. In the interferometric setup, a weak and fast sinusoidal phase modulation $\delta \sin (\omega t)$ is introduced as dither signal, with $\delta=0.4$ radians and $\omega /$ $2 \pi=1600 \mathrm{~Hz}$ (much higher than the inverse of the hologram response time). The dither signal is used to generate (after electronic processing) the voltages $V_{X} \propto \sqrt{\eta} \cos (\varphi-\theta)$ and $V_{Y} \propto \sqrt{\eta} \sin (\varphi-\theta)$, with $\eta$ the diffraction efficiency of the hologram, and $\theta$ an electronically selected phase term that can assume any desired value. For operation, one of these voltages, say $V_{X}$, is used as error signal. As shown in Fig. 2, the error signal is amplified (A) and integrated (INT) to generate the control voltage $V_{C}$, which is amplified (in the power supply of the phase modulator) and fed back to the interferometer. In our setup, a piezoelectrically supported mirror is employed as phase modulator. For operation, a DC bias voltage (not shown in the figure) is added to $V_{C}$ so as to place the mirror somewhere near the middle of its dynamic range (equivalent to about 28 grating periods at $\lambda=532 \mathrm{~nm}$ ). Because of the high gain of the feedback loop, the phase $\varphi$ between the interfering waves is forced to operate around the value that makes $V_{X}=0$, which means $\varphi=\theta \pm 90^{\circ}$, where one of the signs of $\pm 90^{\circ}$ leads to the negative feedback condition. Thus, the loop provides real-time compensation for the phase noise acting in the interferometer, with $\varphi$ controlled through the selection of $\theta$. Because $V_{X}$ operates around zero, $V_{Y}$ assumes its maximum absolute value $\left(V_{Y} \propto \sqrt{\eta}\right)$ and can be used to monitor the diffraction efficiency of the hologram.

\section{Results}

To verify the method we performed stabilized holographic experiments using a nominally undoped $\mathrm{Bi}_{12} \mathrm{TiO}_{20}$ (BTO) crystal. The used sample is known to have electrons as the majority photocarriers at the investigated experimental conditions; this was confirmed by means of the technique originally developed by Orlowski and Krätzig [7], combined with the ellipsometric measurements described by Pauliat et al. [5]. The experiments here reported were carried out at room temperature using the wavelength $\lambda=532 \mathrm{~nm}$ from a linearly polarized solid state laser. The coherent and collimated $R$ and $S$ waves have approximately equal intensities $\left(I_{R} \approx I_{S} \approx 1 \mathrm{~mW} / \mathrm{cm}^{2}\right)$ and intersect symmetrically in the crystal. The grating period is $2 \pi / K=0.64 \mu \mathrm{m}$. The grating wave vector $\mathbf{K}$ lies in the $x$-direction, perpendicular to the crystallographic [001]-axis. The plane containing the grating vector and the [001]-axis corresponds to the (110) crystal face. Silver painted electrodes are deposited on the lateral surfaces of the crystal, which is $4.75 \mathrm{~mm}$ wide (distance between electrodes) and it is $0.95 \mathrm{~mm}$ thick (along the beam direction). Initially, preliminary experiments were performed to find out the feedback condition (phase $\varphi$ ) that led to a stationary hologram in absence of external field; in practice, this was done by simply varying $\theta$ until the control voltage $V_{C}$ became constant over time. The value of $\theta$ (or $\varphi$ ) that produces a stationary hologram depends on some experimental parameters, such as the polarization direction of the input beams and on the detected output waves, but the value itself it is of no importance here. The goal was to record a stationary phase-locked hologram and then apply an electric field in a known direction along the grating wave vector. This procedure is exemplified in Fig. 3A and B. In the first half of the figures there is no external field applied to the sample. The recording is registered from the beginning, with the error signal $V_{X}$ kept around zero due to feedback system. The $V_{Y}$ voltage shows the typical exponential growth of the diffraction efficiency (the time constant is $\approx 2.1 \mathrm{~s}$ ) before the steady-state is reached. Although some small random fluctuations appear in $V_{C}$ (to compensate for environmental phase noise acting in the interferometer) its average value is constant, meaning that the hologram is stationary. The second half of the figures illustrates the recording when the external electric field is applied. Nominally, the field amplitude is $|E|=9.6 \mathrm{kV} / \mathrm{cm}$; as is often pointed out, reduction in the internal field is expected due to various screening effects, and a multiplicative factor of $\approx 0.6$ is usual [29]. The experimental conditions for obtaining the data illustrated in Fig. $3 \mathrm{~A}$ and $\mathrm{B}$ were identical, except for the direction of the external field; it was pointing to the right (positive $x$-direction) in $A$, whereas it was pointing to the left in $\mathrm{B}$. When the field was applied, the diffraction efficiency increased from $\eta \approx 0.5 \%$ to $\approx 1.3 \%$; details on how to compute $\eta$ from $V_{Y}$ (and $V_{D}$ ) are given in Ref. [28]. As discussed earlier, the type of photoconductivity is determined through the slope of $V_{C}$, which indicates that the phase-locked hologram moves in the same direction of the applied field, i.e., to the right in Fig. 3A, and to the left in B. According to Fig. 1 we conclude that electrons are the dominant charge carriers at the studied wavelength and experimental conditions ( $n$-type photoconductivity). As a direct consequence of the results, it is also demonstrated that the hologram moves in the opposite direction to that of the mobile charges; a fact that may not be obvious at a first glance.

As a minor remark, we see on the recordings the appearance of small oscillations in $V_{Y}$ after the field is applied. We believe that the oscillations are most likely due to the existence of a weak photochromic grating recorded in the first half of the experiment. In fact, BTO crystals are known to exhibit light induced absorption effects [3]. Different trap centers may be involved and even though it is a slow process, a modulation in the absorption coefficient of 

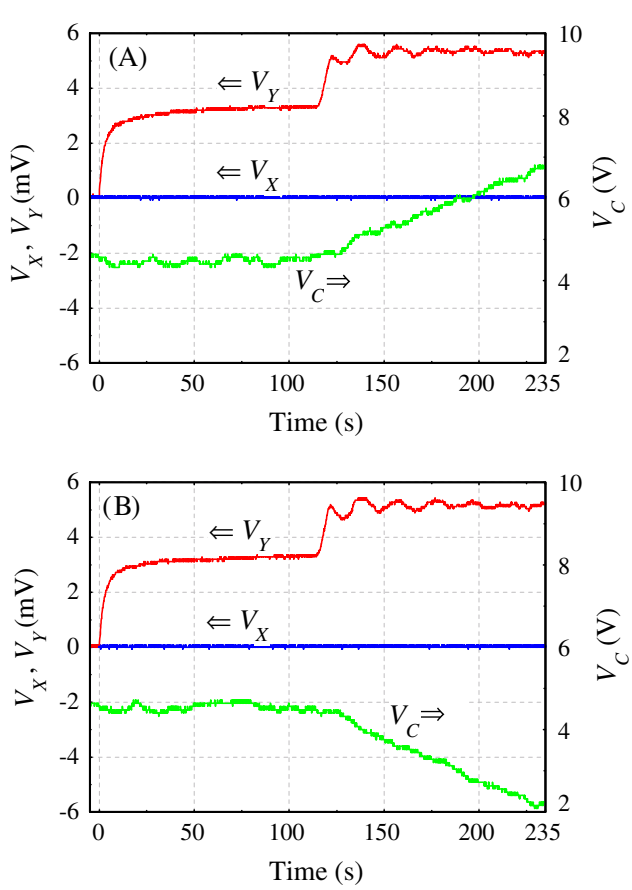

Fig. 3. Experimental results: holographic recording in a BTO crystal under phaselocked regime. The error signal $V_{X}$ operates around zero due to the negative feedback loop, whereas $V_{Y}$ shows the evolution of the hologram diffraction efficiency. In the first half of the figures there is no external electric field applied to the crystal and the hologram is stationary ( $V_{C} \approx$ constant). At the time $t \approx 120 \mathrm{~s}$ the external field is raised from 0 to nominally $9.6 \mathrm{kV} / \mathrm{cm}$. In $(A)$ the field points to the right (positive $x$-direction), whereas in (B) it points to the left. The slope of the control voltage $V_{C}$ is dictated by the field direction and allows determining the sign of the dominant charge carrier (electrons in the present case).

the material should naturally appear if it is exposed to a stationary light pattern for a while. When the light pattern moves it is readily followed by the photorefractive grating, thus producing a beating between the two gratings that lasts until the photochromic grating has vanished. This hypothesis is reinforced by the period of the oscillations (in average about $\approx 19.5 \mathrm{~s}$ ), which is in rather good accordance with the period predicted by the speed of the light pattern, which can be computed from the slope of $V_{C}$ and the known half wave voltage of the phase modulator; the speed is 0.051 grating periods/s in Fig. 3A, and -0.055 grating periods/s in Fig. $3 \mathrm{~B}$.

\section{Discussion}

The results illustrated in Fig. 3A and B were acquired with the photodetector $(D)$ aligned in the $S$ direction (as shown in Fig. 2). Also, the $R$ and $S$ input beams were linearly polarized and oriented to be at $\approx 45^{\circ}$ to the [001]-axis in the middle slice of the sample; this orientation results in parallel-polarized transmitted and diffracted waves at the crystal outputs [30]. In this particular configuration, the phase shift between the interfering output waves that led to a stationary hologram under diffusion regime was $\varphi=0$, which simply means that the waves interfere constructively in the $S$ direction. To check even further the method, a set of experiments were performed with the output light collected in the $R$ direction, as well as for different polarization orientations of the input beams. Just to mention, due to energy conservation reasons, the phase $\varphi$ required to get the stationary hologram condition had sometimes to be changed to $\varphi=\pi$, with the output waves interfering destructively. As expected, in all investigated cases the hologram movement followed the direction of the external applied field.
At this point the reader may have noticed that for the method to work, all we need is to be able to distinguish the change caused by the external field on the slope of the control signal. Thus, although convenient for comparison reasons, the condition of having a stationary hologram before applying the external field is not fundamental. What should be made clear is that the direction of the hologram movement is not enough by itself to determine the sign of the charge carriers, because phase-locked running holograms can be produced even in absence of external field; for ex., by selecting a phase shift $\varphi$ different from the one originated naturally from a stationary light pattern. So, a two-step procedure is required.

We have given above details on the application of the method to the case in which the diffusion is the only internal charge driving force. The extension to the more general case, when both diffusion and photovoltaic driven forces have to be considered is straightforward. The essential difference is that photovoltaic currents take place, which lead to a phase shift $\varphi$ different from 0 or $\pi$ for recording stationary holograms in absence of external field; in fact, when photovoltaic currents largely dominate over diffusion, the phase $\varphi$ approaches to $\pm \pi / 2$ (see chapter 4 of Ref. [25]). In any case, for feedback systems that allow stabilization at arbitrary phase values it is always possible to record a phase-locked hologram by setting properly the control phase of the system; see, for instance, typical regimes of phase-locked holograms recorded in $\mathrm{LiNbO}_{3}$ crystals in Refs. [31,32]. For feedback systems that work only at fixed phase values such as $\varphi=0$ or $\pi$, another approach is to compensate the photovoltaic current by applying an external electric field opposite but equal in magnitude to the photovoltaic saturation field; in this case, only diffusion currents have to be taken into account for the generation of the hologram. Once again, the information on the sign of the main photocarriers can be obtained by observing the effect of an increment of the external applied field on the movement of the phase-locked hologram. Note that investigations on highly insulating crystals (such as weakly doped $\mathrm{LiNbO}_{3}$ ) is not a problem because typical phase noise acting in interferometers (produced by thermal fluctuations, air currents, etc.) is random by nature and has zero average value over long time intervals, thus allowing us to discriminate the influence of the external field on the control voltage $V_{C}$ even if the recording is left for hours or even days.

In summary, holographic feedback techniques have been used for many years as a tool for investigating photorefractive materials. These techniques are able not only to compensate environmental phase noise, but also to shape the characteristics of grating recording and to measure several material parameters. To the best of our knowledge, this is the first paper that describes an experimental procedure for determining the type of photoconductivity in photorefractive materials through feedback systems. This is achieved by a two-step recording process in which an external electric field is further applied to change a stationary phase-locked hologram into a running one. Experimentally, the problem on how to know the movement direction of the hologram is solved by monitoring the slope of the control voltage generated by the system. In our setup, the relation between the voltage slope and the movement direction was previously established by looking directly to the light pattern through a microscope tube; clearly, this relation is a characteristic of the interferometric setup and does not depend on the material. A BTO crystal having n-type photoconductivity was employed to validate the method. Extension of the method to other photorefractive crystals is straightforward.

\section{Acknowledgments}

The authors acknowledge the Brazilian Foundations FAPESP (Fundação de Amparo à Pesquisa do Estado de São Paulo, Brazil) 
and CNPq (Conselho Nacional de Desenvolvimento Científico e Tecnológico, Brazil) for the financial support to this work.

\section{References}

[1] K. Buse, Light-induced charge transport processes in photorefractive crystals I: models and experimental methods, Appl. Phys. B 64 (1997) 273-291.

[2] P. Günter, J.-P. Huignard (Eds.), Photorefractive Materials and Their Applications 3: Applications, Springer Series in Optical Sciences, vol. 115, Springer, New York, 2007.

[3] K. Buse, Light-induced charge transport processes in photorefractive crystals II: materials, Appl. Phys. B 64 (1997) 391-407.

[4] P. Günter, J.-P. Huignard (Eds.), Photorefractive Materials and Their Applications 2: Materials, Springer Series in Optical Sciences, vol. 114 Springer, New York, 2007.

[5] G. Pauliat, M. Allain, J.-C. Launay, G. Roosen, Optical evidence of a photorefractive effect due to holes in $\mathrm{Bi}_{12} \mathrm{GeO}_{20}$ crystals, Opt. Commun. 61 (1987) 321-324.

[6] S.L. Hou, R.B. Lauer, R.E. Aldrich, Transport processes of photoinduced carriers in $\mathrm{Bi}_{12} \mathrm{SiO}_{20}$, J. Appl. Phys. 44 (1973) 2652-2658.

[7] R. Orlowski, E. Krätzig, Holographic method for the determination of photoinduced electron and hole transport in electro-optic crystals, Solid State Commun. 27 (1978) 1351-1354.

[8] A.M. Glass, M.B. Klein, G.C. Valley, Photorefractive determination of the sign of photocarriers in InP and GaAs, Electron. Lett. 21 (1985) 220-221.

[9] A. Donnermeyer, H. Vogt, E. Krätzig, Complementary gratings due to electron and hole conductivity in aluminum-doped bismuth titanium oxide crystals Phys. Status Solidi A 200 (2003) 451-456.

[10] D. Qu, R. Guo, S.M. Liu, Z.H. Liu, Y.M. Gao, Simple optical method for determination of crystal orientation in photorefractive crystals, Appl. Opt. 45 (2006) 6218-6222.

[11] H. Tian, Z. Zhou, D. Gong, H. Wang, Y. Jiang, C. Hou, Photorefractive properties of paraelectric potassium lithium tantalate niobate crystal doped with iron, Opt. Commun. 281 (2008) 1720-1724.

[12] S.L. Sochava, K. Buse, E. Krätzig, Photoinduced hall-current measurements in photorefractive sillenites, Phys. Rev. B 51 (1995) 4684-4686.

[13] A. Gerwens, K. Buse, E. Krätzig, N. Korneev, S. Stepanov, Hall measurements with photorefractive strontium barium niobate by application of a nonsteady-state photocurrent method, J. Opt. Soc. Am. B 15 (1998) 2143-2146.

[14] J.P. Partanen, J.M.C. Jonathan, R.W. Hellwarth, Direct determination of electron-mobility in photorefractive $\mathrm{Bi}_{12} \mathrm{SiO}_{20}$ by a holographic time-of-flight technique, Appl. Phys. Lett. 57 (1990) 2404-2406.

[15] A. Ennouri, M. Tapiero, J.P. Vola, J.P. Zielinger, J.Y. Moisan, J.C. Launay, Determination of the mobility and transport properties of photocarriers in $\mathrm{Bi}_{12} \mathrm{GeO}_{20}$ by the time of flight technique, J. Appl. Phys. 74 (1993) 2180-2191.
[16] A. Ruediger, O. Schirmer, S. Odoulov, A. Shumelyuk, A. Grabar, Studies of lightinduced charge transfer in $\mathrm{Sn}_{2} \mathrm{P}_{2} \mathrm{~S}_{6}$ by combined EPR/optical absorption spectroscopy, Opt. Mater. 18 (2001) 123-125.

[17] S. Juodkazis, V. Netiksis, M. Petrauskas, H.J. Eichler, B. Smandek, Y. Ding, Contactless determination of the dominant photorefractive mobile charge by comparing cw and ps two-wave mixing, Opt. Commun. 134 (1997) 227-232.

[18] L. Boutsikaris, S. Mailis, N.A. Vainos, Determination of the photorefractive parameters of $\mathrm{Bi}(12) \mathrm{SiO}(20)$ by study of the dynamic behavior of complementary gratings, J. Opt. Soc. Am. B 15 (1998) 1042-1051.

[19] N.V. Kukhtarev, V.B. Markov, S.G. Odoulov, M.S. Soskin, V.L. Vinetskii, Holographic storage in electrooptic crystals I: steady state, Ferroelectrics 22 (1979) 949-960.

[20] N.V. Kukhtarev, V.B. Markov, S.G. Odoulov, M.S. Soskin, V.L. Vinetskii, Holographic storage in electrooptic crystals II: beam coupling - light amplification, Ferroelectrics 22 (1979) 961-964.

[21] A.A. Kamshilin, J. Frejlich, L. Cescato, Photorefractive crystals for the stabilization of the holographic setup, Appl. Opt. 25 (1986) 2375-2381.

[22] Ph. Refregier, L. Solymar, H. Rajbenbach, J.P. Huignard, 2-Beam coupling in photorefractive $\mathrm{Bi}_{12} \mathrm{SiO}_{20}$ crystals with moving grating - theory and experiments, J. Appl. Phys. 58 (1985) 45-57.

[23] B.I. Sturman, Space-charge wave effects in photorefractive materials, in: P. Günter, J.-P. Huignard (Eds.), Photorefractive Materials and Their Applications 1: Basic Effects, Springer Series in Optical Sciences, vol. 113, Springer, New York, 2007, pp. 119-162.

[24] A.A. Freschi, P.M. Garcia, J. Frejlich, Phase-controlled photorefractive running holograms, Opt. Commun. 143 (1997) 257-260.

[25] J. Frejlich, Photorefractive Materials, John Wiley \& Sons Inc., Hoboken, New Jersey, 2007.

[26] A.A. Freschi, A.C.C. Telles, J. Frejlich, D.A. Donatti, Oscillating holograms recorded in photorefractive crystals by a frequency detuned feedback loop, J. Appl. Phys. 105 (2009) 023109(1)-023109(7).

[27] E.J. Ambite, L. Arizmendi, Feedback-controlled recording and fixing of photorefractive holograms in reflection geometry on lithium niobate crystals, J. Opt. Soc. Am. B 28 (2011) 1161-1167.

[28] A.A. Freschi, F.S. de Vicente, T.B. Mello, D.A. Donatti, D.R. Vollet, Running holograms in azopolymer films, Appl. Phys. B 111 (2013) 103-109.

[29] A.A. Freschi, P.V. dos Santos, J. Frejlich, Strong and fast phase modulation for quantitative analysis of photorefractive gratings, Appl. Phys. B 83 (2006) 279284.

[30] A. Marrakchi, R.V. Johnson, A.R. Tanguay Jr., Polarization properties of photorefractive diffraction in electrooptic and optically-active sillenite crystals (Bragg regime), J. Opt. Soc. Am. B 3 (1986) 321-336.

[31] A.A. Freschi, Writing saturated holograms in $\mathrm{LiNbO}_{3}$ crystals through phase control and double diffraction, Appl. Phys. B 99 (2010) 487-491.

[32] E.V. Podivilov, B.I. Sturman, S.G. Odoulov, S. Pavlyuk, K.V. Shcherbin, V.Ya. Gayvoronsky, K.H. Ringhofer, V.P. Kamenov, Dynamics of feedback controlled photorefractive beam coupling, Phys. Rev. A 63 (2001) 053805(1)-053805(14). 